Document downloaded from:

http://hdl.handle.net/10251/64026

This paper must be cited as:

Iborra Carreres, A.; Rodríguez Älvarez, MJ.; Soriano Asensi, A.; Sanchez, F.; Bellido, P.; Conde Castellanos, PE.; Crespo Navarro, E.... (2015). Noise Analysis in Computed Tomography (CT) Image Reconstruction using QR-Decomposition Algorithm. IEEE Transactions on Nuclear Science. 62(3):869-875. doi:10.1109/TNS.2015.2422213.

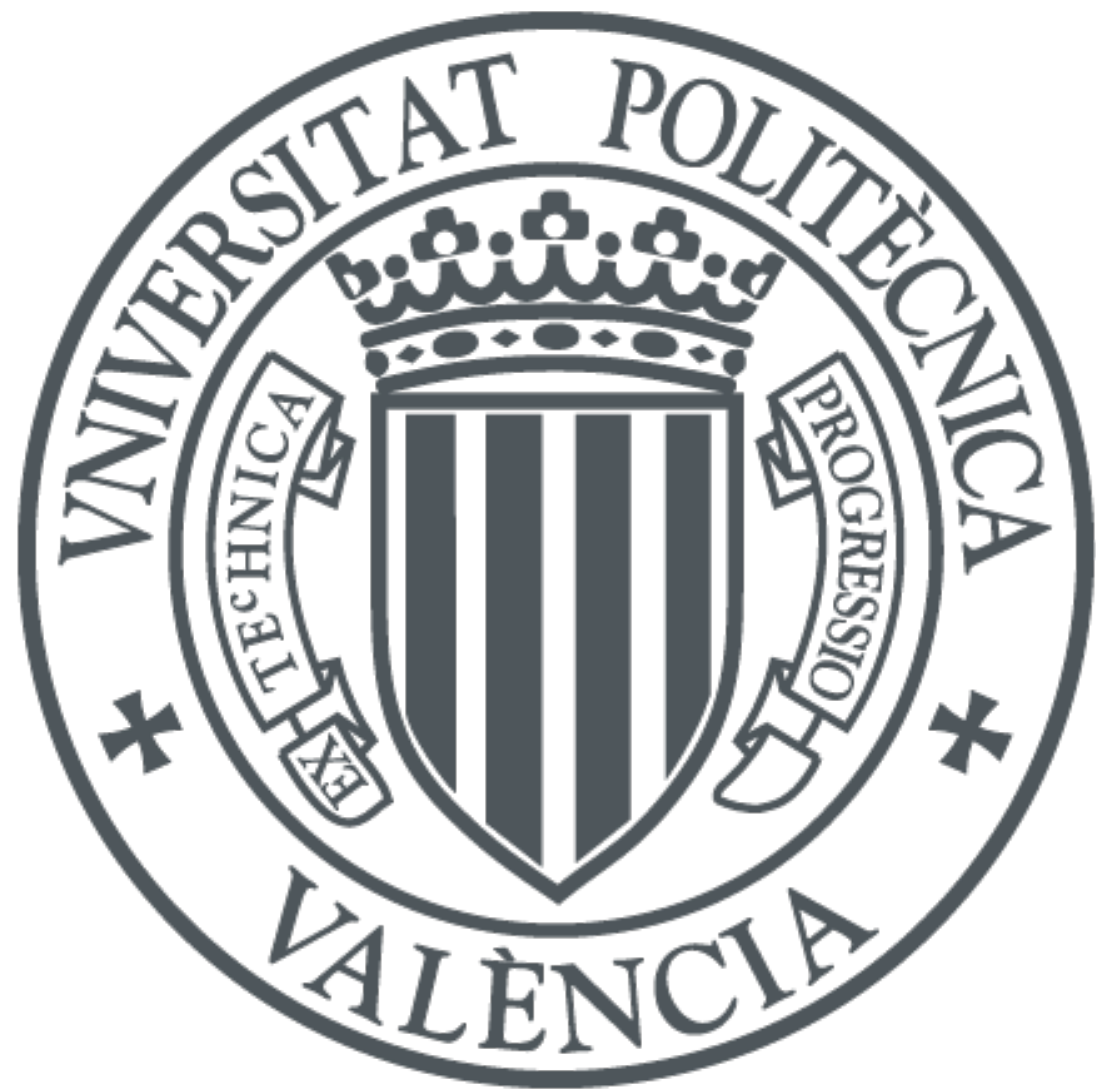

The final publication is available at

http://dx.doi.org/ 10.1109/TNS.2015.2422213

Copyright Institute of Electrical and Electronics Engineers (IEEE)

Additional Information

"C2015 IEEE. Personal use of this material is permitted. Permission from IEEE must be obtained for all other uses, in any current or future media, including reprinting/republishing this material for advertising or promotional purposes, creating new collective works, for resale or redistribution to servers or lists, or reuse of any copyrighted component of this work in other works." 


\title{
Noise analysis in computed tomography (CT) image reconstruction using QR-Decomposition algorithm
}

\author{
A. Iborra*, M. J. Rodríguez-Álvarez, A. Soriano, F. Sánchez, P. Bellido, P. Conde, E. Crespo, A. J. González, \\ L. Moliner, J. P. Rigla, M. Seimetz, L. F. Vidal and J. M. Benlloch
}

\begin{abstract}
The noise of 3D computed tomography (CT) image reconstruction using $Q R$-Decomposition, is analyzed in this paper. There are several types of image noise that can interfere with the interpretation of an image. Here, the noise introduced by the reconstruction process is studied. In this analysis, condition numbers are calculated with different CT model parameters, 3D CT image reconstruction with simulated and real data are performed, image noise analysis is performed through various image quality parameters and the condition number of the linear system is related with the image quality parameters. Results show the condition number's dependence on the CT model. Image reconstructions with simulated data show errors significantly below the condition number theoretical bound and image reconstructions with real data show quality improvement dependence on the condition number. This allows a reduction on the number of projections without compromising image quality and places this reconstruction method as a strong candidate for low-dose 3D CT imaging.
\end{abstract}

Index Terms-Medical imaging, CT low dose imaging, CT image reconstruction, CT modelling, $\mathrm{QR}$ decomposition, image noise.

\section{INTRODUCTION}

$\mathbf{T}$ HE QR-Decomposition algorithm is classified as a model-based $(\mathrm{MB})$ method [1]. MB reconstruction algorithms allow a detailed mathematical description of the physical processes involved in tomographic systems [2], [3]. This advantage can reduce image artifacts and noise and leads toward dose reduction in CT. Model-based iterative reconstruction (MBIR) method is often considered as a general designation for all MB methods [4]. MBIR algorithms arise a large size optimization problem as well as a careful choice of an optimization function along with an iterative search of the solution in which each step involves two matrix vector products. The QR-decomposition algorithm takes advantage of the benefits of the MB approach, but only requires a matrix vector products and backward substitution for the image reconstruction. It could be broadly labeled as a model-based direct reconstruction (MBDIR). QR decomposition process can be computed a priori and it is only necessary to compute them once.

Manuscript received January 1, 1970. This work was supported by the Spanish Plan Nacional de Investigación Científica, Desarrollo e Innovación Tecnológica (I+D+I) under Grant No. FIS2010-21216-CO2-01 and Valencian Local Government under Grants PROMETEOII/2013/010 and ISIC 2011/013.

A. Iborra*, M. J. Rodríguez-Álvarez, A. Soriano, F. Sánchez, P. Bellido, P. Conde, E. Crespo, A. J. González, L. Moliner, J. P. Rigla, M. Seimetz, L. F. Vidal and J. M. Benlloch are with Instituto de Instrumentación para Imagen Molecular (I3M), CSIC - Universidad Politécnica de Valencia CIEMAT, Camino de Vera s/n, Ed. 8-N-1, 46022 Valencia, Spain.

*Corresponding author. Contact: aiborra@i3m.upv.es.
MB methods describe the CT as a linear system of equations $A x=b$, therefore reconstruction problem becomes a linear system where the right hand side $(b)$ corresponds to a vector containing the transmission data (projections) measured by the detectors, and the unknowns $(x)$ correspond to the $3 \mathrm{D}$ image reconstructed, and the matrix $(A)$ describes the CT. A theoretical upper bound of the error that will occur in the reconstruction can be established based on the condition number of the linear system. This bound is defined by the compatibility between the matrix $(A)$ and the right hand side (CT data, $b$ ) of the linear system. In this work we study the relation between this error bound, the reconstructed image quality and the parameters of the CT system. QR decomposition can perform image reconstructions with good quality while using a low number of projections, becoming a candidate for low dose image reconstruction.

The paper is organized as follows. In Section 2, model based image reconstruction is introduced. Measurements and figures of merit are considered in Section 3. In Section 4, results of the analysis of reconstructions are presented. The paper is ended with some concluding remarks in Section 5.

\section{MODEL BASED IMAGE RECONSTRUCTION}

The matrix $A$ models the CT system response in 3D. In a system consisting of $P$ views (projections) measured with a flat-panel divided into $D$ detectors and a field of view (FOV) divided into $N$ voxels, the CT system matrix $A \in \mathbb{R}^{(P D) \times N}$. Each element $a_{i j} \in A$ represents the attenuation on the $i$-th beam caused by the $j$-th voxel. The attenuation is computed as the volume of the intersection between a beam and a voxel. The beam $i$ corresponds with the x-ray beam from the source to a detector $D_{d}$ in a particular view $P_{p}$. In addition, each element $a_{i j}$ is corrected by the cone-beam factor (explained in detail in [5], [6]).

\section{A. QR-Decomposition algorithm}

CT 3D image reconstruction using the QR-Decomposition algorithm is done by the resolution of a linear system of equations

$$
A x=b
$$

using QR-Decomposition with Givens rotations [7], where $A \in \mathbb{R}^{m \times n}$ is the matrix that models the CT system response in $3 \mathrm{D}, b \in \mathbb{R}^{m}$ is the CT measurement and $x \in \mathbb{R}^{n}$ represents the unknown scanned object. Since system (1) 
is overdetermined, the QR solution is equivalent to a least squares problem, fitting solution of (2) [8]

$$
\min \|A x-b\|_{2}
$$

where $\min \|\cdot\|_{2}$ denotes the minimization of the $2-$ norm of the vector $A x-b$. QR-Decomposition is applied to matrix $A$ using Givens rotations, hence the solution of (2) is equivalent to

$$
\min \|Q R x-b\|_{2}
$$

where $Q \in \mathbb{R}^{m \times m}$ is orthogonal and $R \in \mathbb{R}^{n \times n}$ is upper triangular. As $Q$ is orthogonal

$$
\begin{aligned}
\min \|Q R x-b\|_{2} & =\min \left\|Q^{T} Q R x-Q^{T} b\right\|_{2} \\
& =\min \left\|R x-Q^{T} b\right\|_{2}
\end{aligned}
$$

and $x$ (the reconstructed image) is obtained by means of backward substitution process.

\section{$B$. The condition number}

The condition number is an estimator of the sensitivity of a system to perturbations. The definition (5) of the condition number $\left(\kappa_{2}\right)$ usually for a square matrix $A$

$$
\kappa_{2}(A)=\frac{\sigma_{\max }(A)}{\sigma_{\min }(A)}
$$

can be extended to a rectangular matrix [8], [9], [10], where $\sigma_{\max }(A)$ and $\sigma_{\min }(A)$ are the largest and smallest singular values of $A$ respectively. By means of this extension, the condition number of the CT model matrix can be computed and the relative error of the system can be bounded by (6).

$$
\frac{\|\delta x\|_{2}}{\|x\|_{2}} \leq \kappa_{2}(A) \frac{\|\delta b\|_{2}}{\|b\|_{2}}
$$

If $A$ has a low condition number, then low values of $\frac{\|\delta b\|_{2}}{\|b\|_{2}}$ imply low values of $\frac{\|\delta x\|_{2}}{\|x\|_{2}}$ and it is said that the system is well-conditioned. If the condition number is high, then low values of $\frac{\|\delta b\|_{2}}{\|b\|_{2}}$ do not imply low values of $\frac{\|\delta x\|_{2}}{\|x\|_{2}}$ and it is said that the system is ill-conditioned. In other words, the solution of an ill-conditioned system is more sensitive to perturbations in $b$.

\section{MATERIALS AND METHODS}

\section{A. Real CT measurements}

Experimental measurements have been conducted with an Albira $\mu \mathrm{CT}$ [11]. This scanner is a trimodal PET/SPECT/CT. The CT subsystem is a cone beam CT that uses a microfocus $\mathrm{x}$-ray source with a focal spot size of $35 \mu \mathrm{m}$ and a CMOS flat-panel with an active area of $120 \times 120 \mathrm{~mm}^{2}$ that consists of a $2400 \times 2400$ pixelated array sensors.

The phantom used for measurements consists on a polymethylmethacrylate (PMMA) cylinder of $50 \mathrm{~mm}$ height and $55 \mathrm{~mm}$ in diameter and contains cylinders of different materials. The center of each cylinder is $16 \mathrm{~mm}$ off the axis and are $8 \mathrm{~mm}$ in diameter (see figure 1). Aside of the container cylinder of PMMA, the rest of cylinders are of air, polytetrafluoroethylene (PTFE or Teflon), polyethylene (PE)

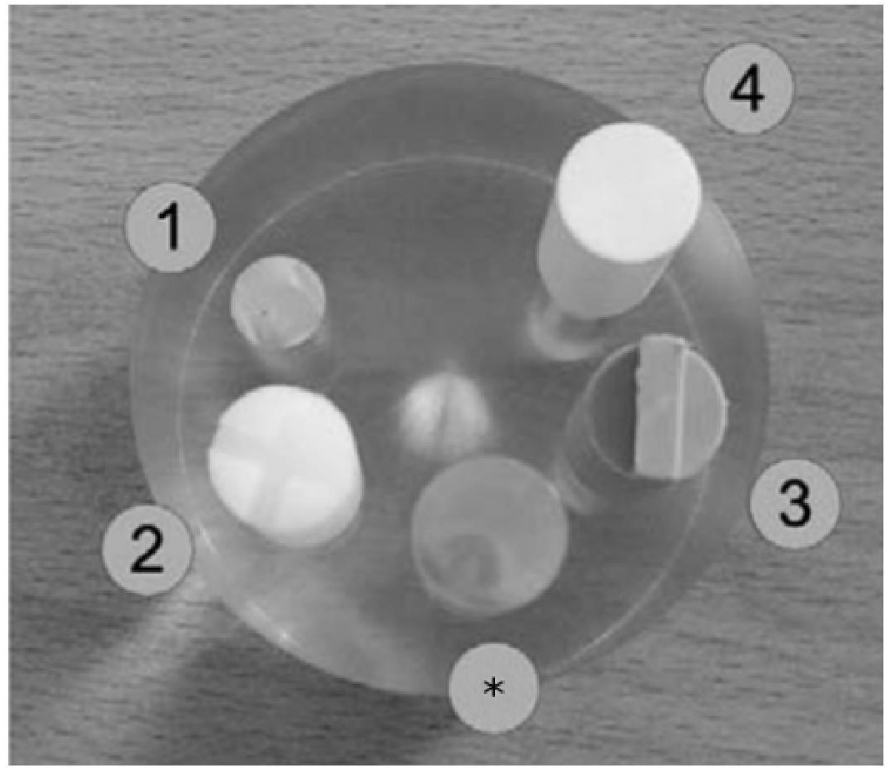

Figure 1. Phantom of PMMA with inserts of PMMA (*) for alignment purposes, air (1), polytetrafluoroethylene (PTFE or Teflon) (2), polyethylene (PE) (3) and polyoxymethylene (POM) (4), which model regions filled with air inside the body, soft bone, adipose tissue, and organs tissue, respectively.

and polyoxymethylene (POM). This materials model regions inside the human body: air regions inside the body, PMMA for soft tissue, PTFE for soft bone, PE for adipose tissue and POM for organs tissue.

Data measurement consisted of 400 projections of the previously described phantom. For measurement, configuration the tube voltage was fixed to $40 \mathrm{kV}$ and $0.5 \mathrm{~mm}$ aluminium filter was used to absorb the lower-energy x-ray photons.

\section{B. Simulated CT measurements}

Using a reconstruction of the CT measurement as a geometrical guide, a 3D image phantom $(x)$ is generated with the expected CT values (depending on the material) in each cylinder. The measurement is modeled as the product of the image phantom and the system matrix. Then, the model measurement is perturbed with several $\delta b$ and used for reconstruction. The model represented by matrix $A$ is an approximation. Certain simplifications were assumed to model the CT system, such as monoenergetic $\mathrm{x}$-ray beams, a punctual $\mathrm{x}$-ray source or the absence of scattering. If $x$ could be scanned with a real CT, $\hat{b}=b+\delta b$ would be obtained. The computed reconstruction $(\hat{x})$ is used to compute the relative error between $x$ and $\hat{x}$. Since the size of $\frac{\|\delta b\|_{2}}{\|b\|_{2}}$ is known the relative error and the condition number can be related.

All CT model configurations have been generated with the geometry of the Albira $\mu \mathrm{CT}$ and therefore can be used for reconstruction with this CT data. The FOV volume is divided into cubic voxels and each voxel will be an element of the reconstructed image.

In this work, we have evaluated the effect of detector size and number of projections on the condition number of the system matrix and the reconstructed image. Different CT model configurations have been generated with a voxel size of $1.28 \mathrm{~mm}$ and varying the other parameters as follows: 
- Detector size: due to computational complexity it is unfeasible to model all hardware detectors. The modeled detectors are a rebinning of the physical detectors. The flat panel area is divided into modeled detectors from $60 \times 60(1.28 \mathrm{~mm}$ detector size $)$ to $192 \times 192(0.4 \mathrm{~mm}$ detector size) in steps of $12 \times 12$ detectors.

- Number of projections: the number of views of the CT measurement along 360 degrees is set from 80 to 300 in steps of 20 projections.

For each combination of these parameters, ten $\delta b$ are randomly generated as a random gaussian distribution to form a set of perturbed measurements. Ten sets are generated with $\frac{\|\delta b\|_{2}}{\|b\|_{2}}$ ranging from $1 \%$ to $10 \%$ in steps of $1 \%$. Then, the relative error in each reconstructed image is computed and averaged among a set to obtain its mean relative error. This perturbation accounts for both physical processes disregarded in the model and finite precision errors or noise in the detection panel.

\section{Figures of merit}

Considering simulated CT measurements, the image quality of the reconstructed image has been evaluated using the relative error (RE).

$$
R E=\frac{\|x-\hat{x}\|_{2}}{\|x\|_{2}}
$$

Considering real CT measurements, the image quality has been evaluated over five volume of interest (VOI) defined by each phantom insert and the PMMA cylinder. For each VOI, a CT number $\left(\mu_{v}\right)$ is computed as the averaged pixel value among the VOI pixels according to the equation (8) and a standard deviation $\left(\sigma_{v}\right)$ as the standard deviation of the pixel values among the VOI pixels according to the equation (9).

$$
\begin{gathered}
\mu_{v}=\frac{1}{N_{v}} \sum_{i=1}^{N_{v}} x_{i} \\
\sigma_{v}=\sqrt{\frac{1}{N_{v}-1} \sum_{i=1}^{N_{v}}\left(x_{i}-\mu_{v}\right)^{2}}
\end{gathered}
$$

where $N_{v}$ is the number of pixels in the VOI and $x_{i}$ is the value of each pixel.

The following image quality indicators have been used:

- Coefficient of variation (CV): is a measure of the differences between pixel values in a VOI. CV is divided by $\mu_{v}$ in order to obtain a dimensionless measure.

$$
C V=\frac{\sigma_{v}}{\mu_{v}} 100
$$

- Contrast recovery coefficient (CRC): is a measure of contrast between a VOI and the PMMA background.

$$
C R C=\frac{\frac{\mu_{v}}{\mu_{b}}-1}{\frac{\mu_{v}^{t}}{\mu_{b}^{t}}-1} 100
$$

where $\mu_{b}$ represents the CT number of PMMA background, $\mu_{v}^{t}$ and $\mu_{b}^{t}$ represents the CT numbers of the materials in the insert and PMMA background, obtained from [12].

- Contrast to noise ratio (CNR): is a measure of the relation between contrast and noise of a VOI and the PMMA background.

$$
C N R=\frac{2\left|\mu_{v}-\mu_{b}\right|}{\sigma_{v}+\sigma_{b}}
$$

where $\mu_{b}$ and $\sigma_{b}$ represent the CT number and the standard deviation of PMMA background respectively.

\section{RESULTS}

This paper shows the impact of the number of projections, and the size of the detectors on the condition number of system matrix of the model and therefore in the noise of reconstructed CT images using the QR-Decomposition method. The optimal values of these two parameters in order to produce wellconditioned models have a linear dependence with the modeled voxel size. The voxel size used in the reconstructions of this work has been $1.28 \mathrm{~mm}$. Similar results were obtained with voxel sizes from $2.13 \mathrm{~mm}$ to $0.91 \mathrm{~mm}$.

\section{A. Model configuration influence on the condition number}

The number of projections, and the size of the detectors for the CT model have a strong influence on the condition number of the resulting system matrix. Figure 2 shows that increasing the number of projections from 100 to 400 with detectors of $0.8 \mathrm{~mm}$ produces a condition number comparable to that obtained with the reduction of the detector size from $0.8 \mathrm{~mm}$ to $0.4 \mathrm{~mm}$ with 100 projections.

Increasing the number of projections and decreasing the detector size produce a growth on the number of equations of the model. As the number of equations grows the condition number tends to stabilize. In this case (with a voxel size of $1.28 \mathrm{~mm}$ ) the condition number stabilizes around $\kappa_{2}(A) \approx 13$ (see figures 2 and 3 ). Although, the condition number is reduced by the number of projections and the detector size, it decreases faster to its limit when the detector size parameter improves.

\section{B. Simulated CT measurements}

The relation between the condition number and the RE of the system is shown in equation (6). Figure 2 shows evolution of the condition number while the number of projections increases. These two curves can be identified in figure 4 for detector sizes of $0.8 \mathrm{~mm}$ and $0.4 \mathrm{~mm}$ respectively. The REs measured tend to the perturbation size $\frac{\|\delta b\|_{2}}{\|b\|_{2}}$, although equation (6) and figures 2 and 3 suggest higher RE values. Similar results were found in all generated $\delta b$.

For a perturbation size of $\frac{\|\delta b\|_{2}}{\|b\|_{2}}=5 \%$ and a RE $\frac{\|\delta x\|_{2}}{\|x\|_{2}} \leq$ $10 \%$, simulated reconstructions with detector sizes below $0.53 \mathrm{~mm}$ and 100 projections or more have obtained lower RE. In fact, simulated reconstruction sets obtained a RE smaller than $2 \frac{\|\delta b\|_{2}}{\|b\|_{2}}$ with detector sizes below $0.53 \mathrm{~mm}$ and 100 projections or more. This suggests that detector sizes below $0.53 \mathrm{~mm}$ and 100 projections can produce images affected by a relatively low degree of error without the need of increasing the number of projections as $\frac{\|\delta b\|_{2}}{\|b\|_{2}}$ increases. 


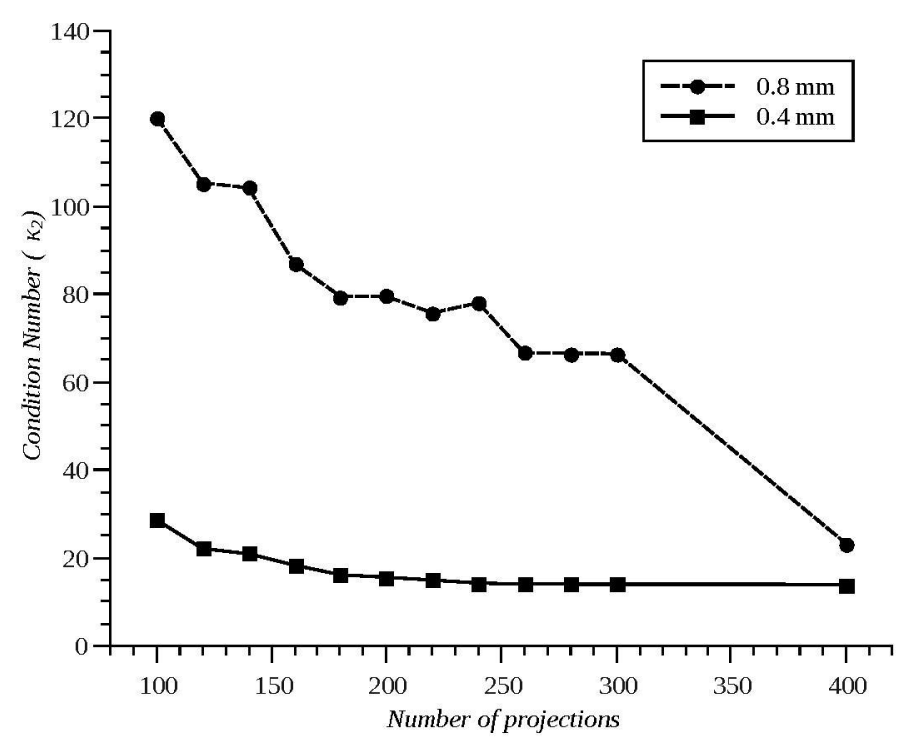

Figure 2. Variation of the condition number of the system matrix as a function of the number of projections. System models have been configured with voxels of $1.28 \mathrm{~mm}$ and with modeled detectors of $0.8 \mathrm{~mm}$ and $0.4 \mathrm{~mm}$.

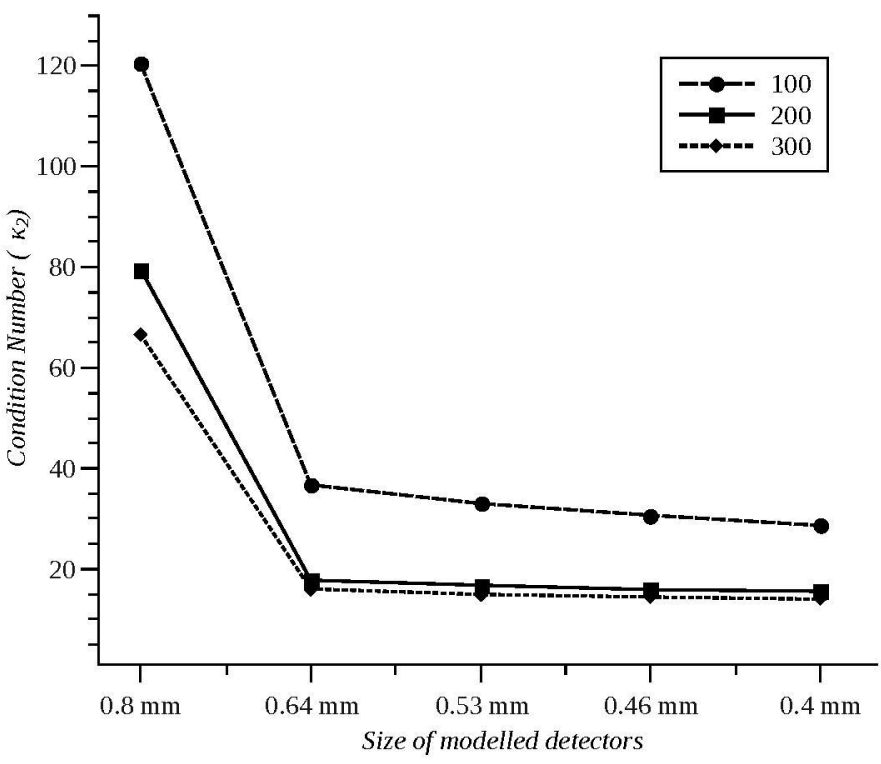

Figure 3. Variation of the condition number of the system matrix as a function of the modeled detector size. System models have been configured with voxels of $1.28 \mathrm{~mm}$ and with 100,200 and 300 projections.

\section{Real CT measurements}

Data measured with Albira $\mu \mathrm{CT}$ of a phantom which models human tissues have been rebined by grouping real detector measurements into modeled detectors of sizes $0.8 \mathrm{~mm}$ and $0.4 \mathrm{~mm}$. The same phantom has been measured along 360 degrees considering 400, 200 and 100 projections. There are 6 compatible model configurations used in the simulated reconstruction compatibles with this data. Transversal slices of 3D reconstructions of these 6 models are shown in figure 6 .

Image quality measurements were performed in phantom VOIs. Results show different levels of relation with the condition number of models.

Regarding $\mathrm{CV}$, the best results have been obtained with

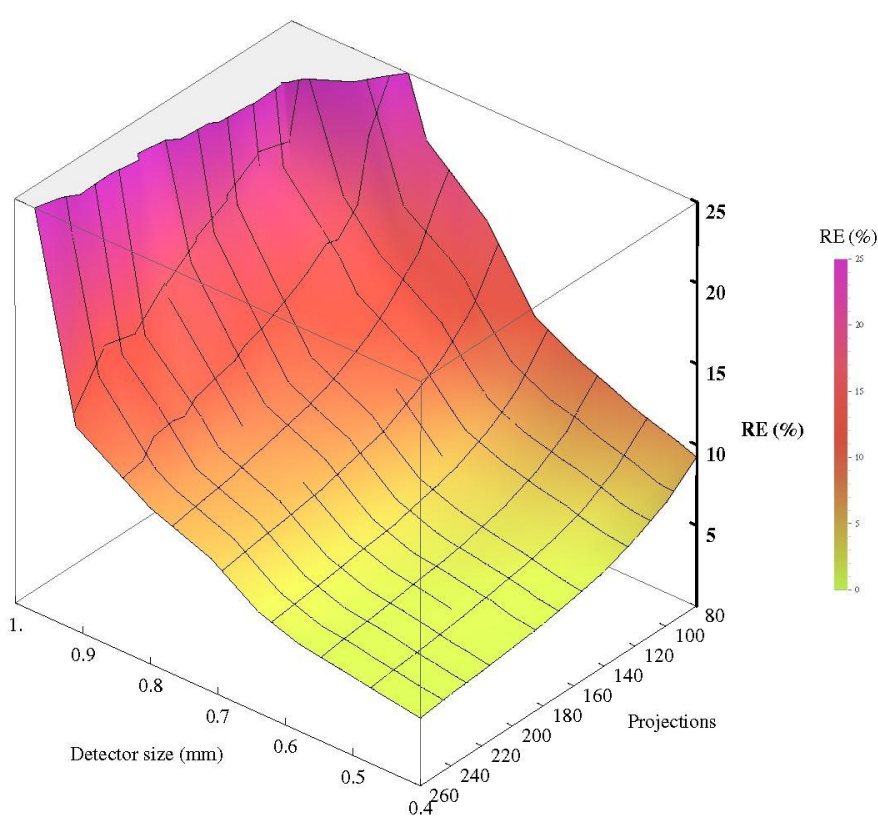

Figure 4. Average REs of image reconstructions of the CT system response model with $\frac{\|\delta b\|_{2}}{\|b\|_{2}}=5 \%$.

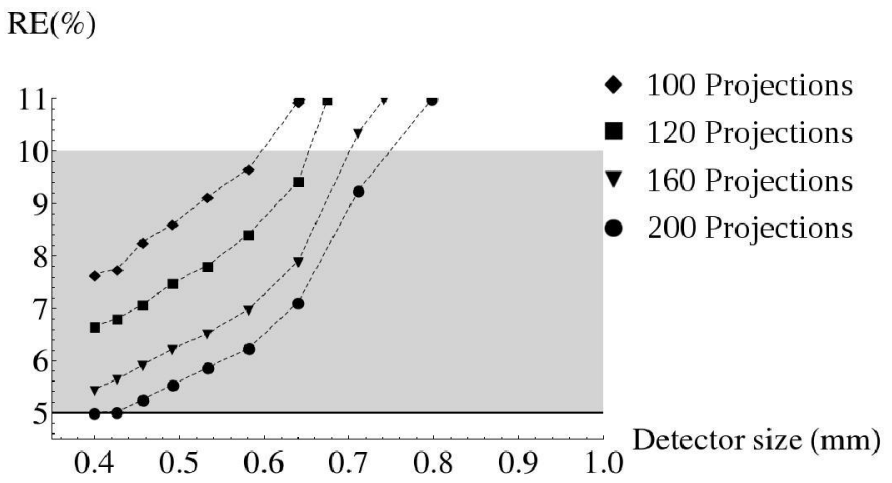

Figure 5. Average REs of image reconstructions for different size of modeled detectors with $\frac{\|\delta b\|_{2}}{\|b\|_{2}}=5 \%$.

the smallest detector sizes. It must be highlighted here that the behavior of the $\mathrm{CV}$ with the increase in the number of projections. There is a notable improvement increasing the number of projections from 100 to 200 and a stabilization from 200 to 400 (see figure 7). In the case of air (see figures 8 and 2) the $\mathrm{CV}$ and the condition number have similar behavior regardless of the number of projections. The small improvement in $\mathrm{CV}$ results from 200 to 400 projections is due to the small improvement on the condition number. Although, the notable improvement in $\mathrm{CV}$ results from 100 to 200 projections is due to the incompleteness of the CT model. As showed in [13], with lower projections, the image CV caused by system modeling error increases and becomes the main source of CV. With 200 projections or more the image CV caused by system modeling error is small compared to other errors such as accumulated round-off error (caused by intermediate 

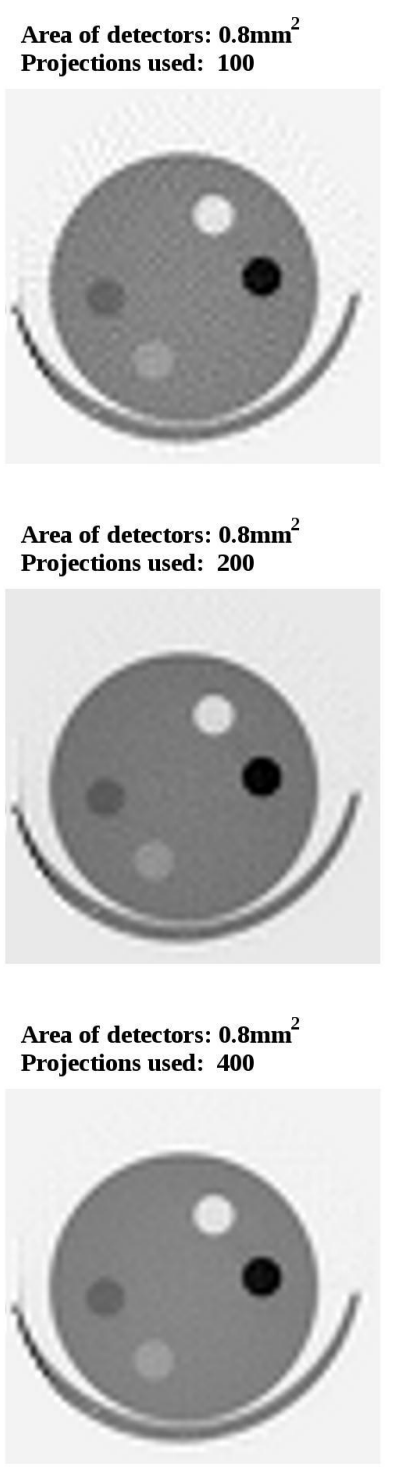

Figure 6. Transversal slice of 3D reconstructions of the measurement performed with Albira $\mu \mathrm{CT}$. Reconstructions of the 6 compatible model configurations are shown. The same gray scale is used for all images. To avoid printing issues, white represents low image values, and black represents high image values.

calculations with floating point precision), numerical stability errors during the system matrix decomposition or noise in CT flat panel measurement.

Furthermore, special mention should be made of the CV of air (see figure 8). CV values of air are ten times larger than the rest of VOIs. This is caused by low values of $\mu_{\text {air }}$, which are around 0.05 . Although, values of $\sigma_{\text {air }}$ are between 0.02 and 0.008 , which are similar to the other VOIs as PMMA.

As for CRC, the best results have been obtained with the smallest detector sizes. However, differences between models are lower than $1 \%$, regardless of the CT number of VOIs (see figure 9). With such varying conditions, as from 100 to 400 projections or detector sizes from $0.8 \mathrm{~mm}$ to $0.4 \mathrm{~mm}$, QRDecomposition reconstruction produces images with stable contrast relations.

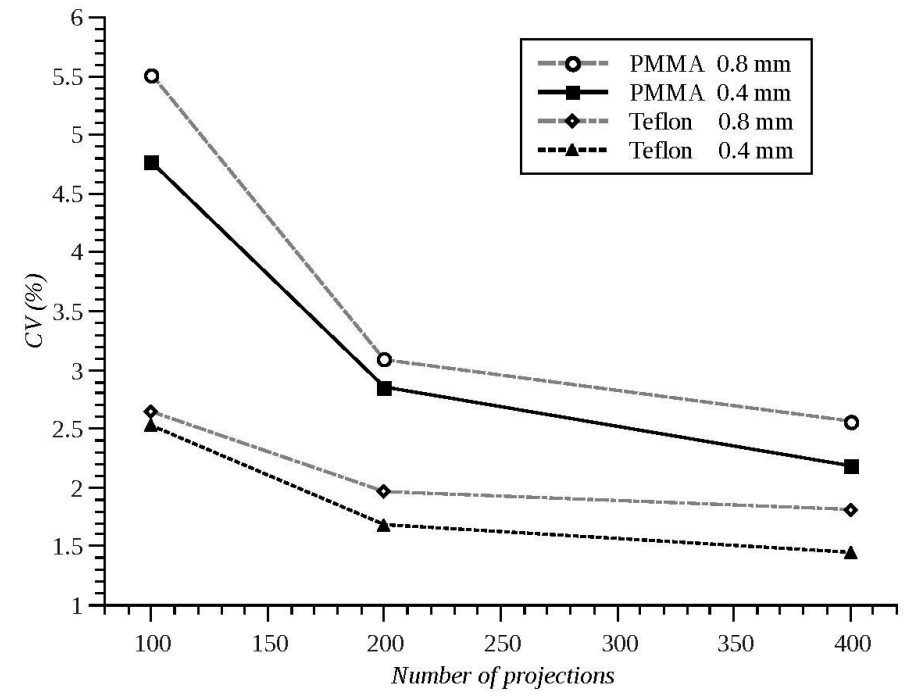

Figure 7. Coefficient of variation of Teflon and PMMA in the reconstructed images with Albira $\mu \mathrm{CT}$ data as number of projection increases. Systems with modeled detector sizes of $0.8 \mathrm{~mm}$ and $0.4 \mathrm{~mm}$ are shown.

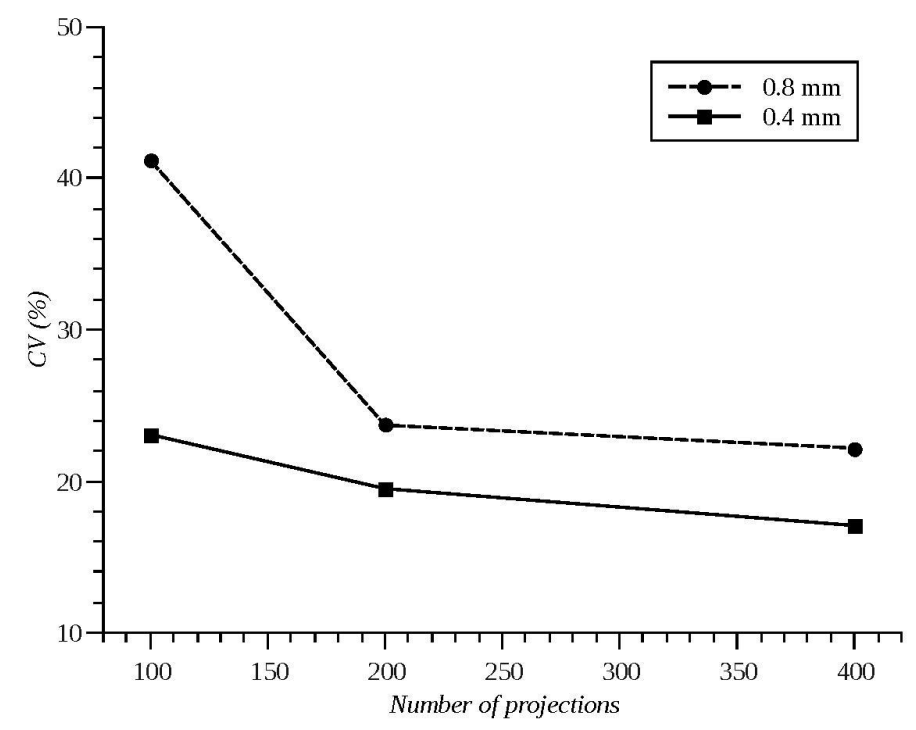

Figure 8. Coefficient of variation of air in reconstructed images with Albira $\mu \mathrm{CT}$ data as number of projection increases. Systems with modeled detector sizes of $0.8 \mathrm{~mm}$ and $0.4 \mathrm{~mm}$ are shown.

Regarding CNR, the best results have been obtained with the improvement of both number of projections and detector size. Although this parameter obtains the highest improvements as the number of projections increases (around 15\%) also improves with the decreasing of the detector size (between $2 \%$ and $8 \%$, see figure 10). The same reason as in CV, incompleteness of the CT model relates this parameter with the number of projections.

\section{CONCLUSIONS}

In this work a MBDIR algorithm is used to reconstruct CT images. The parameters of the CT model influence the condition number of the matrix used to reconstruct 3D images. The parameter which influences the condition number the most is the detector size. The reconstruction of simulated data 


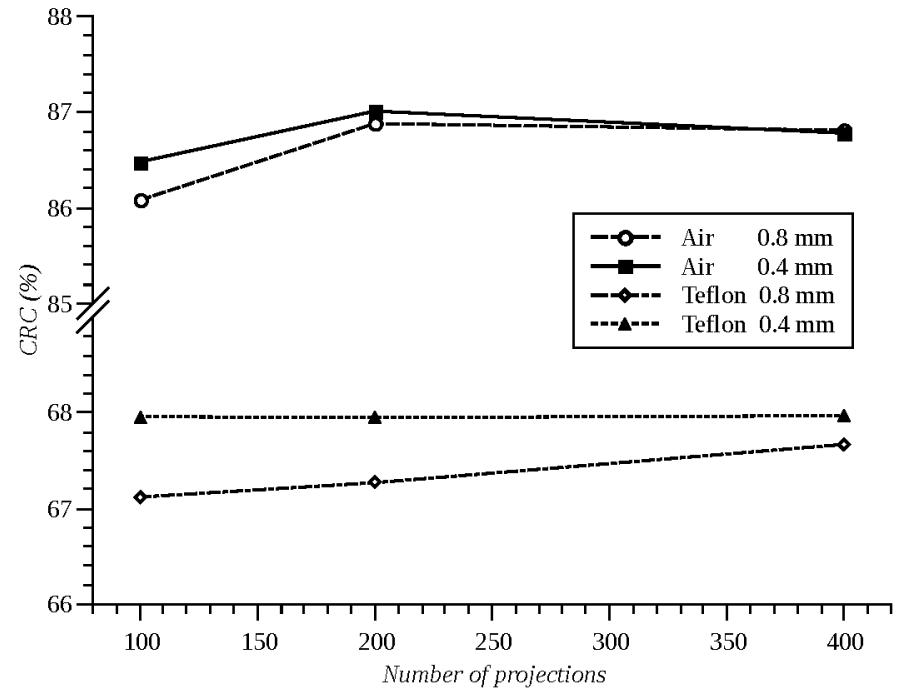

Figure 9. Contrast recovery coefficient of Teflon and air in reconstructed images with Albira $\mu \mathrm{CT}$ data as number of projection increases. Systems with modeled detector sizes of $0.8 \mathrm{~mm}$ and $0.4 \mathrm{~mm}$ are shown.

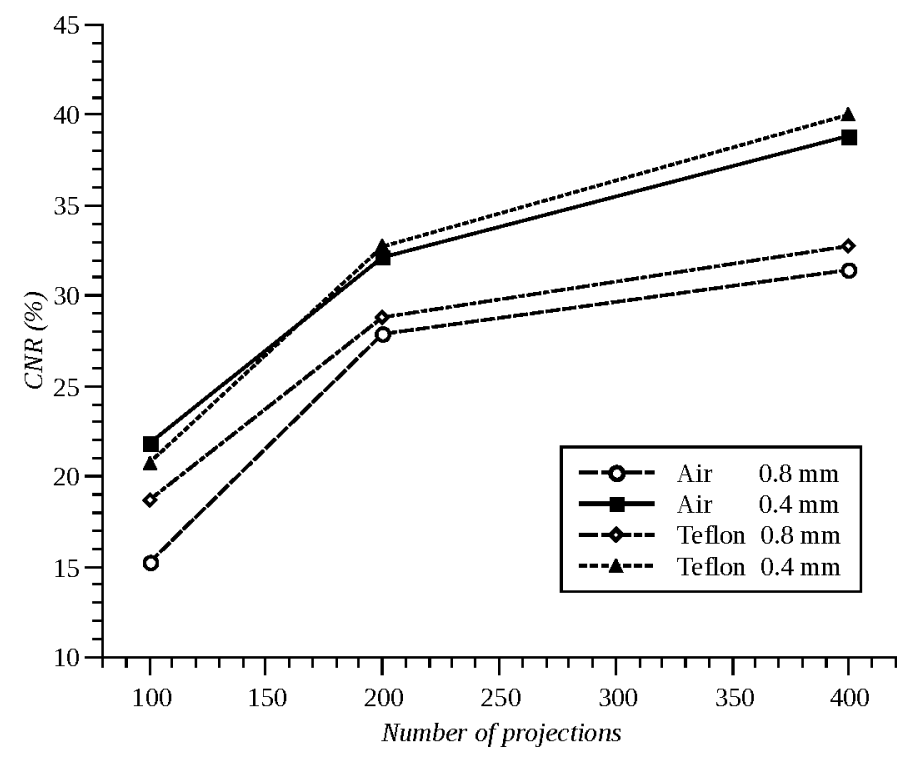

Figure 10. Contrast to noise ratio of Teflon and air in reconstructed images with Albira $\mu \mathrm{CT}$ data as number of projection increases. Systems with modeled detector sizes of $0.8 \mathrm{~mm}$ and $0.4 \mathrm{~mm}$ are shown.

showed that the improvement of the condition number of the system has an impact on the RE of the reconstructed images beyond the theoretical bound of equation (6).

Image quality measurements of reconstructed images using the same CT models with data from Albira $\mu \mathrm{CT}$ showed that:

- CRC of VOIs remains constant (around 1\% of variation) regardless of the parameters chosen (size of modeled detectors between $0.8 \mathrm{~mm}$ and $0.4 \mathrm{~mm}$ and number of projections between 100 an 400 ).

- CV of VOIs improves mainly with the number of projections from 100 to 200 and for more than 200 projections, major improvement is related to detector size.

- CNR of VOIs improves with the combination of number of projections and the size of the detectors. The relation between the CNR and the number of projections is due to the incompleteness of the CT model.

CRC stability and $\mathrm{CV}$ improvement as detector size decreases, enables a potential reduction on the number of projections used for the image reconstruction. This reduction on the number of projections implies the reduction of radiation dose received by the patient.

The reason of grouping physical detectors in larger modeled detectors and disregard physical processes in the CT model is the ease of computational complexity. Improvements on the decomposition of the system matrix will allow to model smaller detectors that will imply better condition numbers and produce reconstructed images with better quality parameters without the need of a large number of projections. For these reasons we propose the 3D image reconstruction using $\mathrm{QR}$ Decomposition as a promising candidate for CT low-dose imaging.

\section{REFERENCES}

[1] M. Beister, D. Kolditz, and W. A. Kalender, "Iterative reconstruction methods in X-ray CT.," Physica medica : PM : an international journal devoted to the applications of physics to medicine and biology : official journal of the Italian Association of Biomedical Physics (AIFB), vol. 28, pp. 94-108, Apr. 2012.

[2] M.-J. Rodríguez-Álvarez, F. Sánchez, A. Soriano, A. Iborra, and C. Mora, "Exploiting symmetries for weight matrix design in CT imaging," Mathematical and Computer Modelling, vol. 54, no. 7-8, pp. 1655-1664, 2011.

[3] J. Hsieh, B. Nett, Z. Yu, K. Sauer, J.-B. Thibault, and C. Bouman, "Recent Advances in CT Image Reconstruction," Current Radiology Reports, vol. 1, pp. 39-51, Jan. 2013.

[4] Z. Yu, J,-B. Thibault, C. A. Bouman, K. D. Sauer, and J. Hsieh, "Fast model-based X-ray CT reconstruction using spatially nonhomogeneous ICD optimization.," IEEE transactions on image processing : a publication of the IEEE Signal Processing Society, vol. 20, pp. 161-75, Jan. 2011.

[5] W. Yao and K. Leszczynsky, "Analytically derived weighting factors for transmission tomography cone beam projections," Physics in medicine and biology, vol. 54, no. 3, pp. 513-533, 2009.

[6] M. J. Rodríguez-Álvarez, A. Soriano, A. Iborra, F. Sánchez, A. J. González, P. Conde, L. Hernández, L. Moliner, A. Orero, L. F. Vidal, and J. M. Benlloch, "Expectation maximization (EM) algorithms using polar symmetries for computed tomography (CT) image reconstruction," vol. 43, no. 8, pp. 1053-1061, 2013.

[7] M.-J. Rodríguez-Álvarez, F. Sánchez, A. Soriano, and A. Iborra, "Sparse Givens resolution of large system of linear equations: Applications to image reconstruction," Mathematical and Computer Modelling, vol, 52, no. 7-8, pp. $1258-1264,2010$.

[8] G. H. Golub and C. F. V. Loan, Matrix Computations. JHU Press, 1996.

[9] D. S. Watkins, Fundamentals of Matrix Computations. John Wiley and Sons. Inc., 2002.

[10] B. Li and Z. Jia, "Some results on condition numbers of the scaled total least squares problem," Linear Algebra and its Applications, vol. 435, pp. 674-686, Aug. 2011.

[11] F. Sánchez, A. Orero, A. Soriano, C. Correcher, P. Conde, A. González, L. Hernández, L. Moliner, M. J. Rodríguez-Álvarez, L. F. Vidal, J. M. Benlloch, S. E. Chapman, and W. M. Leevy, "ALBIRA: A small animal PET/SPECT/CT imaging system," Medical Physics, vol. 40, no. 5, p. 051906, 2013.

[12] J. H. Hubbell and S. M. Seltzer, "Tables of X-Ray Mass Attenuation Coefficients and Mass Energy-Absorption Coefficients from $1 \mathrm{keV}$ to $20 \mathrm{MeV}$ for Elements $Z=1$ to 92 and 48 Additional Substances of Dosimetric Interest." NIST Standard Reference Database 126, 2004.

[13] J. Qi and R. H. Huesman, "Effect of Errors in the System Matrix on MAP Image Reconstruction," Physics in medicine and biology, vol. 50, pp. 3297-3312, July 2005. 